\title{
Charmonium studies at Belle
}

\author{
Kirill Chilikin ${ }^{1, *}$ \\ ${ }^{1}$ Lebedev Physical Institute of the Russian Academy of Sciences, Moscow
}

Abstract. A review of recent charmonium studies at Belle is presented.

\section{Introduction}

Several studies of conventional charmonium states of candidates have been performed recently by the Belle Collaboration. A review of these studies is presented here. It includes the observation of the $\chi_{c 0}$ (3860) [1], measurement of the absolute $B^{+} \rightarrow X_{c \bar{c}} K^{+}$branching fractions [2], observation of $e^{+} e^{-} \rightarrow \gamma \chi_{c 1}$ [3], and evidence for $B^{+} \rightarrow h_{c} K^{+}$, that has been found in the same analysis with the observation of $\eta_{c}(2 S) \rightarrow p \bar{p} \pi^{+} \pi^{-}$[4].

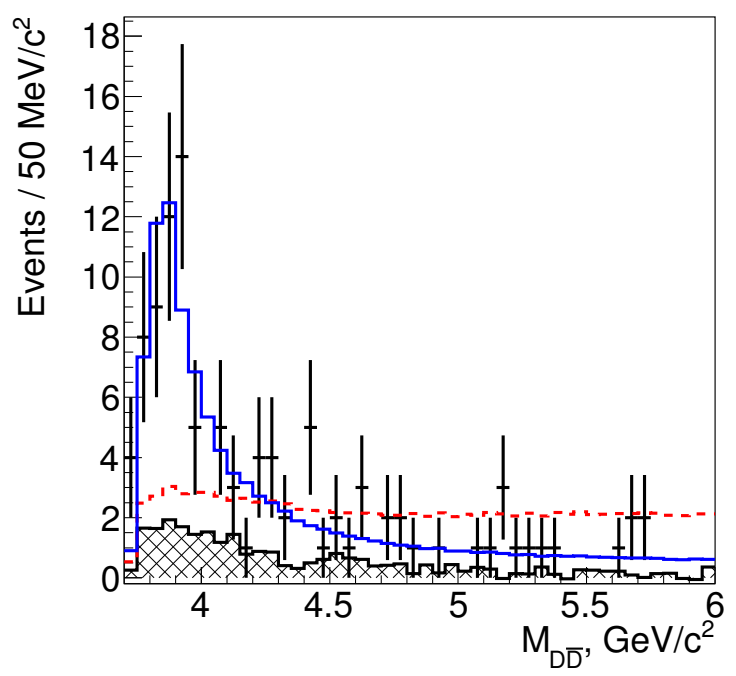

Figure 1. Projections of the $e^{+} e^{-} \rightarrow J / \psi D^{(*)} \bar{D}^{(*)}$ fit results onto $M_{D \bar{D}}$. The points with error bars are the data, the hatched histogram is the background, the blue solid line is the fit with the $X^{*}$ resonance $\left(J^{P C}=\right.$ $\mathrm{O}^{++}$), and the red dashed line is the fit with nonresonant amplitude only. Figure from reference [1].

*e-mail: chilikin@lebedev.ru 


\section{Analyses}

\subsection{Observation of an alternative $\chi_{c 0}(2 P)$ candidate}

The $\chi_{c 0}(3860)$ was observed in the process $e^{+} e^{-} \rightarrow J / \psi D \bar{D}[1]$, where $D$ means either $D^{0}$ or $D^{+}$. The event selection was optimized to improve the sensitivity. A multivariate analysis was performed for each $D$ decay channel. After that, a global optimization of the selection requirements has been performed, which included the channel-specific definitions of the signal region and the neural-network output requirements.

The default model of the process $e^{+} e^{-} \rightarrow J / \psi D \bar{D}$ included the nonresonant amplitude (with three different parameterizations) and an additional resonance $X^{*}$ decaying to $D \bar{D}$. The $X^{*}$ produced in this process has even spin and positive parity; the $C$-parity should also be positive. Thus, two quantum-number hypotheses were considered: $J^{P C}=0^{++}$and $2^{++}$. A new charmoniumlike state $X^{*}(3860)$ was observed with a significance of $6.5 \sigma$. The preferred $X^{*}$ (3860) quantum-number hypothesis was found to be $J^{P C}=0^{++}$(thus, it can also be named the $\chi_{c 0}(3860)$ ), however, the $2^{++}$hypothesis was not excluded: the $0^{++}$hypothesis is preferred at $2.5 \sigma$ level. The $\chi_{c 0}(3860)$ parameters were measured to be $M=3862_{-32-13}^{+26+40} \mathrm{MeV} / c^{2}$ and $\Gamma=201_{-67-82}^{+154+88} \mathrm{MeV}$. Projections of the fit results onto $M_{D \bar{D}}$ in the models with and without the $\chi_{c 0}(3860)$ are shown in figure 1.

The new $\chi_{c 0}(3860)$ resonance seems to be a better candidate for the $\chi_{c 0}(2 P)$ charmonium state than the $X(3915)$, because its properties are well matched to expectations for the $\chi_{c 0}(2 P)$ resonance. Such properties include the mass, the mass difference with the $\chi_{c 2}(2 P)$, the largewidth $D \bar{D}$ observation decay mode, the production that is consistent with pure $S$-wave as is the case for the process $e^{+} e^{-} \rightarrow J / \psi \chi_{c 0}(1 P)$. In addition, the $\chi_{c 0}(3860)$ mass and width agree with the $\chi_{c 0}(2 P)$ parameters determined from an alternative fit to the Belle and BaBar $\gamma \gamma \rightarrow D \bar{D}$ data [5].

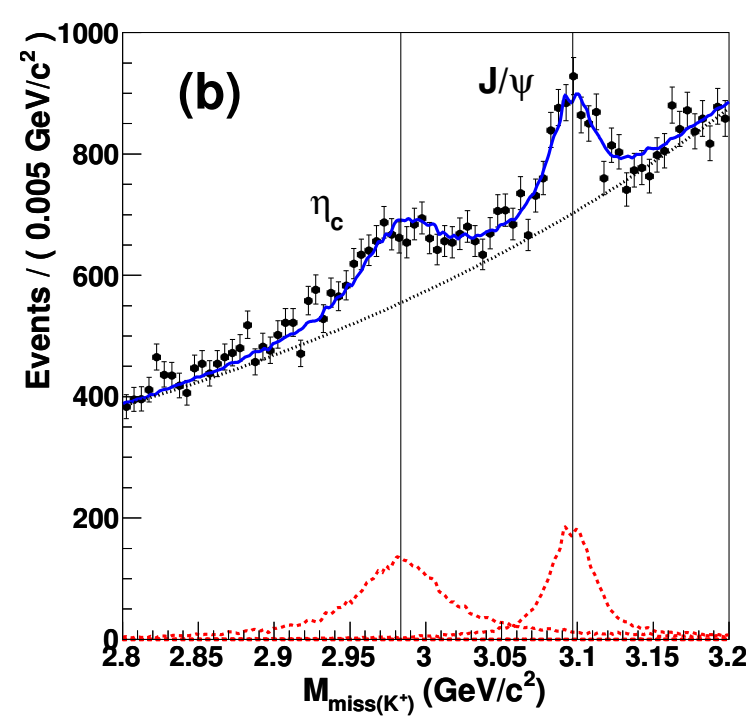

Figure 2. Missing-mass distribution in the region of the $\eta_{c}$ and $J / \psi$. The points with error bars are data, the blue solid line is the fit result, the grey dotted line is the background component, and the red dashed lines are the $\eta_{c}$ and $J / \psi$ signals Figure from reference [2]. 


\subsection{Absolute branching fractions of $B^{+} \rightarrow X_{c \bar{c}} K^{+}$}

Absolute branching fractions of the decays $B^{+} \rightarrow X_{c \bar{c}} K^{+}$, where $X_{c \bar{c}}$ denotes $\eta_{c}, J / \psi, \chi_{c 0}, \chi_{c 1}$, $\eta_{c}(2 S), \psi(2 S), \psi(3770), X(3872)$, and $X(3915)$, and also $B^{+} \rightarrow \pi^{+} D^{(*) 0}$, were measured [2] using the missing-mass distribution with a full reconstruction of one of the $B$ mesons and a kaon from another one. The tagging $B$-meson was reconstructed in one of 1104 hadronic decays using a hierarchical hadronic full reconstruction algorithm based on the NeuroBayes neural-network package. No significant signal for $X(3872)$ was observed; an upper limit of $\mathcal{B}\left(B^{+} \rightarrow X(3872) K^{+}\right)<2.6 \times 10^{-4}(90 \%$ C.L.), which is more stringent than the previous one determined by BaBar [6] $\left(3.2 \times 10^{-4}\right)$. The upper limit of $\mathcal{B}\left(B^{+} \rightarrow X(3915) K^{+}\right)<2.8 \times 10^{-4}$ ( $90 \%$ C.L.) has been set for the first time. The resulting branching fractions $\mathcal{B}\left(B^{+} \rightarrow \eta_{c} K^{+}\right)=$ $(12.0 \pm 0.8 \pm 0.7) \times 10^{-4}$ and $\mathcal{B}\left(B^{+} \rightarrow \eta_{c}(2 S) K^{+}\right)=(4.8 \pm 1.1 \pm 0.3) \times 10^{-4}$ are the most accurate measurements to date. In particular, this is the first significant measurement for $\mathcal{B}\left(B^{+} \rightarrow \eta_{c}(2 S) K^{+}\right)$. The uncertainty of the current world average of $\mathcal{B}\left(\eta_{c}(2 S) \rightarrow K \bar{K} \pi\right.$ [7] is dominated by the measurement of $\mathcal{B}\left(B^{+} \rightarrow \eta_{c}(2 S) K^{+}\right)=(3.4 \pm 1.8) \times 10^{-4}$ by BaBar [6]; the new measurement significantly improves the precision of $\mathcal{B}\left(\eta_{c}(2 S) \rightarrow K \bar{K} \pi\right)$.

2.3 Observation of $e^{+} e^{-} \rightarrow \gamma \chi_{c 1}$

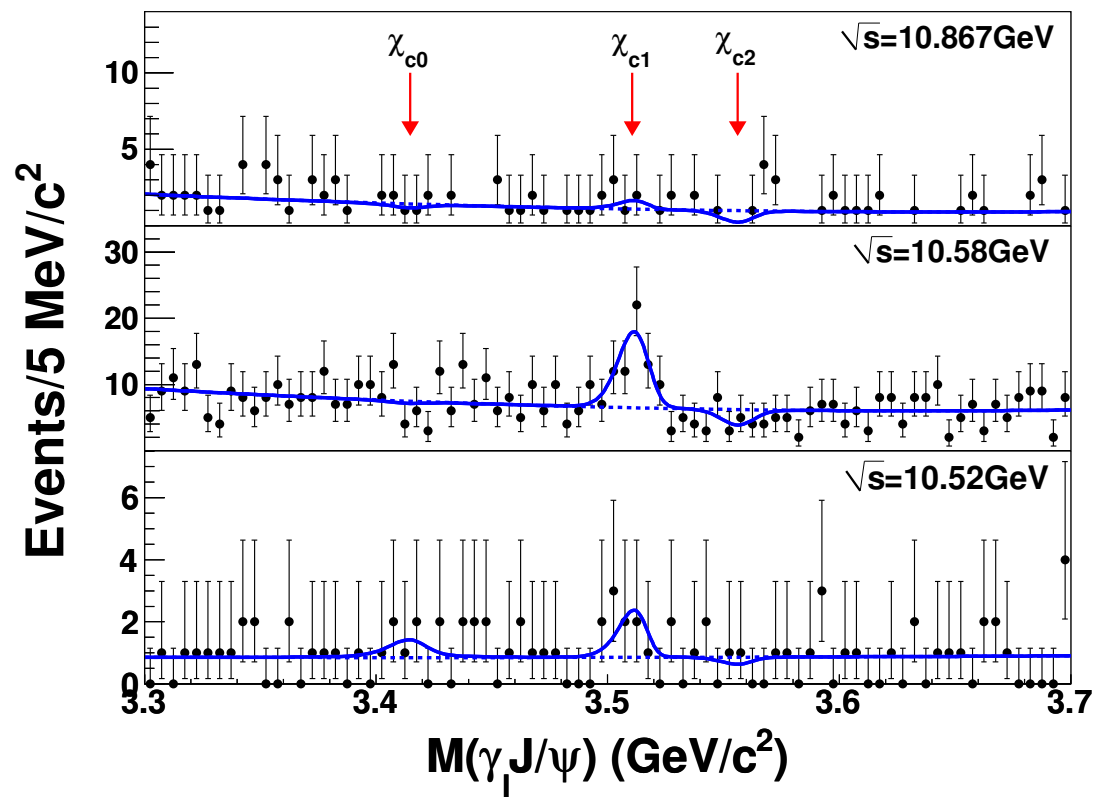

Figure 3. The $J / \psi \gamma$ invariant mass spectra at $\sqrt{s}=10.52$ (bottom), 10.58 (middle), and $10.867 \mathrm{GeV}$ (top) together with the fit results. The points with error bars are data, the blue solid lines are the fit results, and the blue dashed lines are the backgrounds contributions. The arrows show the masses of the $\chi_{c 0}, \chi_{c 1}$, and $\chi_{c 2}$ states. Figure from reference [3].

A search for the process $e^{+} e^{-} \rightarrow \gamma \chi_{c J}(J=0,1,2)$ and $\gamma \eta_{c}$ has been performed at $\sqrt{s}=$ $10.52 \mathrm{GeV}, 10.58 \mathrm{GeV}$, and $10.867 \mathrm{GeV}$ using a $922 \mathrm{fb}^{-1}$ data sample [3]. The $\chi_{c J}$ mesons were reconstructed using their decay $\chi_{c J} \rightarrow J / \psi \gamma$ with $J / \psi \rightarrow \mu^{+} \mu^{-}$; the $\eta_{c}$ candidates were reconstructed in five hadronic decays: $K_{S}^{0} K^{+} \pi^{-}, \pi^{+} \pi^{-} K^{+} K^{-}, 2\left(\pi^{+} \pi^{-}\right), 2\left(K^{+} K^{-}\right)$, and $3\left(\pi^{+} \pi^{-}\right)$. A clear $\chi_{c 1}$ signal was observed at $10.58 \mathrm{GeV}$ with a statistical significance of $5.2 \sigma$, and the 
Born cross section was measured to be $\left(17.3_{-3.9}^{+4.2}\right.$ (stat.) \pm 1.7 (syst.)) fb. No significant signal was found for $\chi_{c 0} \gamma, \chi_{c 2} \gamma$, and $\eta_{c} \gamma$ final states. For the cases where a $\chi_{c J}$ or $\eta_{c}$ signal is not evident, upper limits on the Born cross sections were determined at $90 \%$ C.L. Using the cross sections measured at three different $\sqrt{s}$ in this analysis and from BESIII at much lower $\sqrt{s}$ and assuming the reaction $e^{+} e^{-} \rightarrow \gamma \chi_{c 1}$ proceeds through the continuum process only, the cross section $s$-dependence has been determined to be $1 / s^{2.1_{0.4}^{+0.3} \pm 0.3}$ for $e^{+} e^{-} \rightarrow \gamma \chi_{c 1}$.

\subsection{Evidence for $B^{+} \rightarrow h_{c} K^{+}$and observation of $\eta_{c}(2 S) \rightarrow p \bar{p} \pi^{+} \pi^{-}$}

A search for the decays $B^{+} \rightarrow h_{c} K^{+}$and $B^{0} \rightarrow h_{c} K_{S}^{0}$ has been performed [4]. The integrated luminosity unsed for analysis is 2.8 times greater than the luminosity used in the previous Belle analysis [8]. For further improvement of the sensitivity, the new analysis used ten $\eta_{c}$ decay channels to reconstruct the decay $h_{c} \rightarrow \eta_{c} \gamma$; only two channels were used in the old one. The new $h_{c}$ decay channel $h_{c} \rightarrow p \bar{p} \pi^{+} \pi^{-}$observed recently by the BESIII Collaboration [9] was also used for its reconstruction; in addition, the decays of other charmonium states to $p \bar{p} \pi^{+} \pi^{-}$were studied. The discrimination of the signal and background events was improved by performing a multivariate analysis. An automated optimization of the selection criteria (the channel-dependent two-dimensional $\left(\Delta E, M_{b c}\right)$ signal regions and requirements on the neural-network output) was performed.

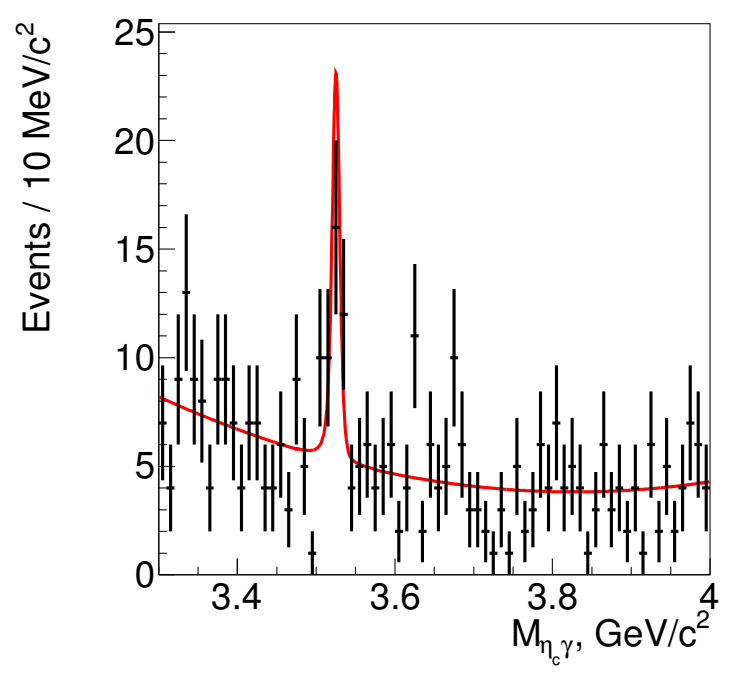

Figure 4. Fit results in the $h_{c} \rightarrow \eta_{c} \gamma$ signal for the $B^{+} \rightarrow h_{c} K^{+}$channel. The points with error bars are data and the red solid line is the fit result. Figure from reference [4].

A simultaneous extended unbinned maximum likelihood fit to the $h_{c} \rightarrow \eta_{c} \gamma$ signal, $h_{c} \rightarrow$ $p \bar{p} \pi^{+} \pi^{-}$background, and $h_{c} \rightarrow p \bar{p} \pi^{+} \pi^{-}$signal distributions has been performed. The fit results for the decay $B^{+} \rightarrow h_{c} K^{+}$are shown in Figure 4 for the $h_{c} \rightarrow \eta_{c} \gamma$ signal and in Figure 5 for the $h_{c} \rightarrow p \bar{p} \pi^{+} \pi^{-}$signal. The significance of the decays $B^{+} \rightarrow h_{c} K^{+}$and $B^{0} \rightarrow h_{c} K_{S}^{0}$ with the systematic error taken into account was found to be $4.8 \sigma$ and $0.7 \sigma$, respectively. Thus, evidence for the decay $B^{+} \rightarrow h_{c} K^{+}$has been found, but no evidence was found for 
$B^{0} \rightarrow h_{c} K_{S}^{0}$. The significance of the $\eta_{c}(2 S) \rightarrow p \bar{p} \pi^{+} \pi^{-}$was measured to be $12.1 \sigma$ and $5.8 \sigma$ for the processes $B^{+} \rightarrow(c \bar{c})\left(\rightarrow p \bar{p} \pi^{+} \pi^{-}\right) K^{+}$and $B^{0} \rightarrow(c \bar{c})\left(\rightarrow p \bar{p} \pi^{+} \pi^{-}\right) K_{S}^{0}$, respectively. The significance including the systematic error is $12.1 \sigma$ and $5.8 \sigma$, respectively. Consequently, the decay $\eta_{c}(2 S) \rightarrow p \bar{p} \pi^{+} \pi^{-}$was observed for the first time in both $B^{+} \rightarrow(c \bar{c})\left(\rightarrow p \bar{p} \pi^{+} \pi^{-}\right) K^{+}$ and $B^{0} \rightarrow(c \bar{c})\left(\rightarrow p \bar{p} \pi^{+} \pi^{-}\right) K_{S}^{0}$ processes.
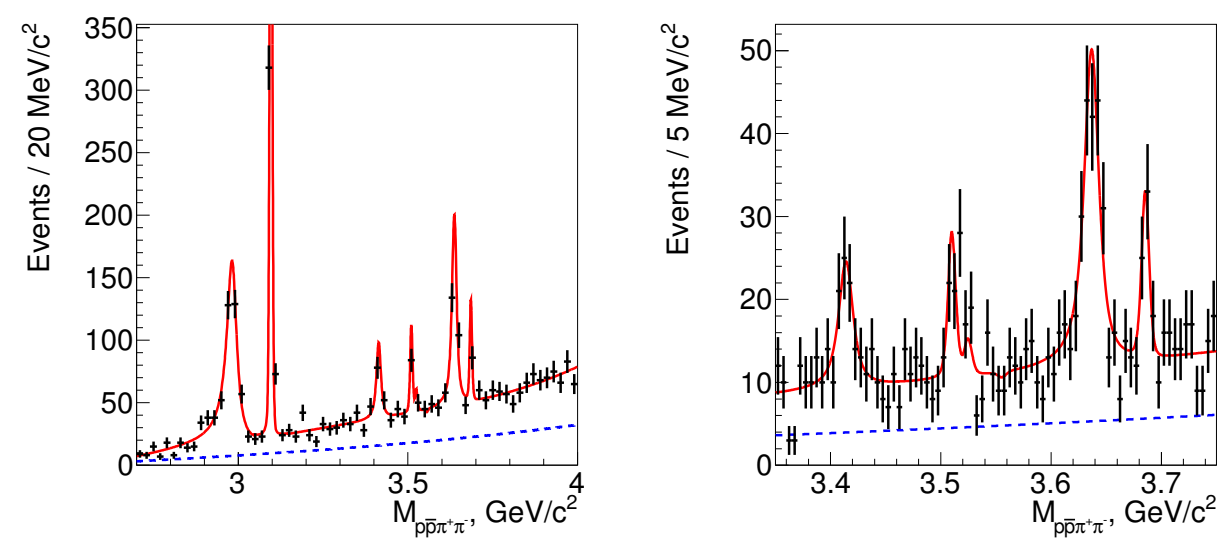

Figure 5. Fit results in the $h_{c} \rightarrow p \bar{p} \pi^{+} \pi^{-}$signal in the entire fitting region (left) and in the $\chi_{c J}$ region (right) for the $B^{+} \rightarrow h_{c} K^{+}$channel. The points with error bars are data, the red solid line is the fit result, and the blue dashed line is the background. Figures from reference [4].

The branching fractions of the decays $B^{+} \rightarrow h_{c} K^{+}$and $B^{0} \rightarrow h_{c} K_{S}^{0}$, as well as the branching fraction products $\mathcal{B}\left(B^{+} \rightarrow(c \bar{c}) K^{+}\right) \times \mathcal{B}\left((c \bar{c}) \rightarrow p \bar{p} \pi^{+} \pi^{-}\right)$and $\mathcal{B}\left(B^{0} \rightarrow\right.$ $\left.(c \bar{c}) K_{S}^{0}\right) \times \mathcal{B}\left((c \bar{c}) \rightarrow p \bar{p} \pi^{+} \pi^{-}\right)$were measured. The branching fraction of $B^{+} \rightarrow h_{c} K^{+}$was found to be $\left(3.7_{-0.9-0.8}^{+1.0+0.8}\right) \times 10^{-5}$; the upper limit for the $B^{0} \rightarrow h_{c} K_{S}^{0}$ branching fraction is $1.4 \times 10^{-5}$ at $90 \%$ C. L.

\section{References}

[1] K. Chilikin et al. (Belle Collaboration), Phys. Rev. D 95, 112003 (2017).

[2] Y. Kato et al. (Belle Collaboration), Phys. Rev. D 97, 012005 (2018).

[3] S. Jia et al. (Belle Collaboration), Phys. Rev. D 98, 092015 (2018).

[4] K. Chilikin et al. (Belle Collaboration), arXiv:1903.06414 [hep-ex].

[5] F. K. Guo and U. G. Meissner, Phys. Rev. D 86, 091501 (2012).

[6] B. Aubert et al. (BaBar Collaboration), Phys. Rev. Lett. 96, 052002 (2006).

[7] M. Tanabashi et al. (Particle Data Group), Phys. Rev. D 98, 030001 (2018).

[8] F. Fang et al. (Belle Collaboration), Phys. Rev. D 74, 012007 (2006).

[9] M. Ablikim et al. (BESIII Collaboration), Phys. Rev. D 99, 072008 (2019). 